\title{
Un acercamiento al presente, pasado y futuro de la geografía aplicada en Iberoamérica
}

\section{An approach to the past, present and future of applied geography in Ibero-America}

\author{
Carlos Garrocho Rangel, Gustavo D. Buzai (20i 5 ) (coords.), \\ GEOGRAFÍA APLICADA EN IBEROAMÉRICA. AVANCES, RETOS Y PERSPECTIVAS, \\ El Colegio Mexiquense, A. C., Toluca, 588 Pp., ISBN 978-607- \\ 776I-76-I.
}

El libro que se reseña reúne a un grupo de investigadores de alto nivel cuyos estudios se enfocan en temas y problemas de Iberoamérica; cuentan con un perfil de ciencias sociales espacialmente integradas, como lo mencionan los coordinadores de este libro. En México, se habla de investigación socioespacial, que no es más que el cruce de todas las ciencias sociales (sociología, economía, antropología, geografía...) con las llamadas ciencias exactas (matemáticas, física, computación, ingenierías...). La integración de ciencias es actualmente una de las dinámicas del mundo científico, en la cual se vislumbra un panorama de alto potencial hacia el futuro.

La obra incluye a veintitrés autores de diversas instituciones en Iberoamérica. Se divide en tres ejes principales, el primero es el radar geográfico, es decir, una aproximación que examina la geografía social, económica y ambiental; el segundo, está integrado por temas que estudian el cruce de las ciencias sociales espacialmente integradas: terminología, análisis espacial, tecnologías vinculadas a la información geográfica; el tercero, lo componen investigaciones aplicadas, donde se presentan ejemplos de investigación sobresaliente en Iberoamericana.

Los trabajos que integran los capítulos se enfocan en las ciencias sociales que le otorgan un papel estratégico a la dimensión espacial, registrando un enorme interés en el mundo, e Iberoamérica no escapa a esta tendencia. Esto se puede observar en la abundante producción científica de la región, derivada de este enfoque, en sus contribuciones claves al diseño de políticas públicas y acciones privadas o en la consolidación de numerosas revistas especializadas como EURE, en Chile, y Economía, Sociedad y Territorio, en México.

Los autores muestran una fascinación por la investigación socioespacial, la cual sitúa los procesos sociales en el territorio, que explora la relación 
mutua entre lo social y lo espacial a diversas escalas geográficas en el tiempo, esto ha generado un boom tanto en el ámbito académico como en las esferas pública, privada y social. Afirman que en el mundo del siglo XXI, las aplicaciones geográficas, inclusive, en teléfonos celulares, son de uso cotidiano para gran parte de la sociedad (GPS o Google Maps, por ejemplo) con evolución nunca antes vista en la historia.

El libro abre con una introducción extensa, pero precisa sobre su contenido, en ésta Carlos Garrocho Rangel, investigador de El Colegio Mexiquense, expone los puntos cardinales de la geografía aplicada en el siglo XXI, asimismo a partir de un ejemplo realiza un análisis socioespacial de la segregación de adultos mayores.

En el capítulo de Priscilla Connolly, de la Universidad Autónoma Metropolitana-Azcapotzalco, se identifican los principales enfoques teóricos de los estudios urbano-regionales en América Latina durante las pasadas cinco décadas, y perfila las posibles rutas al futuro. Muestra que los estudios urbano-regionales registran una notable diversificación temática y metodológica de los enfoques disciplinares de la geografía en el nuevo siglo.

El segundo capítulo escrito por Josep Maria Arauzo Carod, de la Universitat Rovira i Virgili e Institud d'Economia de Barcelona, Cataluña, se enfoca en mostrar la evolución de la geografía económica, fundamentalmente en Cataluña. Este tema es un punto cardinal en la geografía del siglo XXI por su relación multidisciplinaria, donde el tema central de investigación es la aglomeración de las actividades económicas.

Por su parte, Boris Graizbord, investigador de El Colegio de México realiza una destacada participación en el libro a partir de una amplia revisión de la geografía ambiental. Muestra que lo innovador en una propuesta científica resulta de la intersección o combinación de al menos dos disciplinas o subdisciplinas, a lo que llama hibridación en la geografía. Menciona que son múltiples las ciencias sociales que integran a esta disciplina, donde retoman la importante pregunta ¿¿dónde?, cuestión que conduce a la consideración de diversos elementos centrales de esa disciplina. Destaca que los procesos ambientales interactúan con procesos sociales y crean interrelaciones multidiplinares a escalas diferentes.

En el capítulo cuatro, Lorenzo López Trigal, de la Universidad de León, España, hace una reflexión del lenguaje científico de la geografía y propone contar con una terminología geográfica, es decir, "un lenguaje especializado". Tarea poco sencilla ya que la sistematización de cualquier terminología científica es dinámica en cualquier disciplina pues es notable la renovación terminológica y temática de la producción científica.

Gustavo D. Buzai, de la Universidad Nacional de Luján, Argentina, continúa con el tema de la llamada nueva geografía, también llamada 
geografía teórica, geografía estadística o macrogeografia (cap. 5). Lo anterior es alimentado por diferentes tendencias y paradigmas que abarcan desde el manejo cuantitativo hasta cuestiones locacionales, humanísticas o críticas. Donde menciona que la geografía se entiende como la disciplina que introduce el espacio como dimensión de los fenómenos físicos y sociales.

Los vínculos entre geografía aplicada y tecnologías de la información son prácticamente inevitables en el siglo XXI, porque han transformado por completo la pregunta fundamental ¿cómo? Con ello los geógrafos y otros especialistas adquieren y generan conocimiento socioespacial. Esta discusión es propuesta por Ernest Ruiz i Almar, de la Universidad de Barcelona, donde menciona "actualmente es posible representar y comunicar ordenada, eficiente y fácilmente ideas territoriales complejas de una manera compresible y transformar simples datos espaciales en información geográfica con los cuales se puede extraer conocimientos acerca del territorio y de la sociedad" (p. 65).

Por su parte Claudia Baxendale, de la Universidad de Buenos Aires, Argentina, analiza las principales articulaciones conceptuales entre la geografía como ciencia pura y aplicada, el ordenamiento territorial y los sistemas de información geográfica, como práctica y relevancia central en la geografía. Este capítulo es la cara opuesta del análisis socioespacial, observa que la geografía se ha desvirtuado e identifica diversas contradicciones que explican este proceso: las contradicciones entre ciencia, tecnología y práctica territorial. Mientras que deja ver que la geografía ha intensificado su focalización en objetos de estudio no espaciales, por ejemplo: actores o agentes sociales, imágenes mentales de lugares o conductas en lugares determinados.

Siguiendo la secuencia del libro, el capítulo ocho muestra ejemplos de trabajos de investigación en geografía aplicada y se organiza en torno a tres ejes claves: geografía social, geografía económica y geografía ambiental. Este capítulo realizado por Guillermo Velázquez, de la Universidad Nacional del Centro de la Provincia de Buenos Aires, Argentina, trata sobre la calidad de vida en Argentina (geografía social), desde una perspectiva geográfica y analiza los cambios en la calidad de vida de la población sudamericana en el presente siglo. Apoyándose en SIG, muestra nuevos métodos en la geografía aplicada que es una introducción a la multidisciplina.

En el capítulo nueve, escrito por Alejandro Salazar Burrows, de la Pontifica Universidad Católica de Chile, se analizan las recomposiciones socioterritoriales en los espacios perimetropolitanos y su significado para las regiones urbanas en América Latina. Destaca la importancia de los espacios, el autor los llama perimetropolitanos; muestra nuevas rutas de 
investigación y aplicación de la ciencia geográfica. Se identifica que uno de los objetivos es diseñar planes o estrategias socioterritoriales en múltiples escalas para que se adecuen a ciudades latinoamericanas.

El capítulo 10, a cargo de Djamel Toudert de El Colegio de la Frontera Norte, habla de la vinculación entre la brecha digital y la marginación socioterritorial para el caso de México. Menciona que la construcción de la sociogeografía es una compleja matriz de conocimientos de distintas disciplinas. Esto lo traduce en la capacidad de acomodar armónicamente conocimiento multi, inter y trans-disciplinario, además de llevarlo a una traducción de métodos estadísticos avanzados.

Renato Miguel do Carmo, del Instituto Universitario de Lisboa, comienza con las contribuciones próximas a la geografía económica (cap. 11). Explora las relaciones entre las poblaciones rural y urbana a partir del principio de cooperación, muestra cómo son las interrelaciones rururbanas (i. e. son las competencias cooperativas y la confianza), encaminado al diseño de políticas públicas. El objeto del capítulo es mostrar las características asimétricas en diversas variables de estudio.

El capítulo 12 es una aportación conjunta de dos investigadores situados en dos países Iberoamericanos, Antonio Moreno, de la Universidad Autónoma de Madrid, y Manuel Fuenzalida, de la Universidad Alberto Hurtado, Chile. Parten de la interrogante ¿quién consigue qué, cómo, cuánto, cuándo, dónde?, pregunta clásica de la geografía, enfocada en los servicios y equipamiento para la población en España y Chile. Donde adoptan los conocimientos de otras disciplinas como el marketing y la psicología del consumidor. Además, disponen de geodatos y combinan modelos de localización con técnicas de evaluación multicriterio.

La colaboración de varios investigadores como Richard Shearmur, de la Universida de McGill, Canadá; Carlos Garrocho, José Antonio Álvarez y Tania Chávez, de El Colegio Mexiquense, México, analizan la geografía de las actividades económicas en la Ciudad de México, al considerar no sólo métodos y conceptos, sino al incorporar dimensiones de alta complejidad: cultura y subjetividad. Con el propósito de explorar en qué medida diversos patrones de la globalización de la región norte son útiles para analizar la geografía económica de ciudades latinoamericanas (cap. 13).

El capítulo 14, escrito por Miguel Ángel García López e Iván Muñiz Olivera, de la Universidad Autónoma de Barcelona, devela la estructura espacial de la población y la infraestructura de transporte en Barcelona. El objetivo es analizar el papel de diferentes infraestructuras de transporte como autopistas, carreteras principales y ferrocarriles, que conforman la estructura espacial de la población en la metrópoli de Barcelona.

En el capítulo 15, escrito por Eduardo Salinas Chávez y Ricardo Remond, de la Universidad de la Habana, enfatizan el enfoque integrador 
de la Geografía del paisaje en los estudios territoriales aplicados en el siglo XXI. Mencionan que el proceso de desarrollo es largo y complejo de integrar a los estudios de geografía en América Latina. Analizan dos países (Cuba y Ecuador), para demostrar los estudios de ordenamiento, planificación y gestión territorial a diferentes escalas geográficas.

Adriana Piperno y Pablo Sierra, de la Universidad de la Republica, Uruguay, escriben el capítulo 16, centrando su atención en las ciudades y adoptando un enfoque integral. Los autores toman la gestión del territorio, como ejemplo el agua, incluyendo el análisis de la multiplicidad de la escala, siendo posible su abordaje como gran cuenca territorial (suelo productivo) hasta las microcuencas urbanas (drenaje).

Los coordinadores del libro, Gustavo Buzai y Carlos Garrrocho, determinan los puntos más importantes de la nueva geografía aplicada. El primero de los puntos es el acceso instantáneo del conocimiento multi, inter y trans-disciplinario que puede ser acomodado coherentemente en la Geografía. El segundo punto son los nuevos métodos de análisis espacio temporal. El tercero es la disponibilidad de grandes bases de información (nunca antes vistas en la historia) y, el cuarto, es el desarrollo tecnológico, principalmente programas de cómputo y la capacitación de individuos para su manejo.

Para cerrar el libro, Gustavo Buzai hace una reflexión sobre el nuevo camino de la geografía aplicada. Advierte que en el siglo XXI existen posibilidades técnicas excelentes para la creación y distribución de datos espaciales de utilidad para la geografía aplicada. La desventaja es que la fluidez y disponibilidad de los datos varían entre países, estas diferencias son más visibles en países latinoamericanos.

El análisis a diferentes escalas, la realidad del territorio se encuentra a diversos niveles de análisis y procesos, por ello se genera el uso de teorías en sus niveles específicos, con lo que quedan delimitados los niveles $\mathrm{N}+1$, $\mathrm{N}$ y N-1 (así los define el autor). En nivel focal $\mathrm{N}$ es donde vive el análisis espacial de la geografía como la aplicamos. El nivel N+1 llamado supranivel y el nivel N-1 llamado infranivel, propiciaran el contexto necesario para la aplicación de otras teorías y el uso de otros conceptos.

La tecnología actual brinda la posibilidad de la generación de datos geográficos masivos y, por tanto, su análisis. Estamos en la etapa en la que los conocimientos científicos comienzan a vislumbrar como práctica social por medio del uso tecnológico que tiene más componentes basados en la localización. Aparece la Neogeografia a partir de la cual la sociedad podrá colaborar en la generación de datos y procedimientos geográficos.

La geografía aplicada es el primer paso en la realización de estudios concretos uniendo diferentes disciplinas, esto no garantiza la transferencia de resultados. La separación entre procedimientos de planificación y 
gestión delimita claramente hasta dónde pueden llegar los estudios científicos y desde dónde el poder político-administrativo tiene responsabilidades en cuanto al uso de la ciencia.

Resumiendo, el contenido del libro queda explicado en su título, objeto, enfoque y estructura, sobre todo, queda establecido el tono que dominará en el futuro de la geografía aplicada. Claridad en la exposición, transparencia en las intenciones, declarada aspiración a la profundidad y ánimo de discusión, sin elusión de la controversia.

Los primeros siete capítulos del libro se basan en la definición de la geografía aplicada en el siglo XXI. Destaca la participación de Boriz Graizbord, proponiendo el término hibridación de la ciencia (combinación y recombinación entre varias disciplinas), por medio de la geografía ambiental llega a dar una explicación clara y coherente de esta combinación científica.

Realizan un recorrido por la historia de la geografía y proponen el camino que llevará a la geografía aplicada en el siglo XXI. Los capítulos restantes tienen tres ejes principales en los cuales se expone la explicación del uso de la nueva geografía: geografía social, geografía económica y geografía ambiental.

En conclusión, el libro capta una diversidad de factores históricos que examinan el problema de la geografía. La percepción de la nueva geografía se compara con las semejanzas de esta disciplina en el siglo XX. Desafortunadamente no hay tantas semejanzas debido a un factor muy importante que los autores llaman humanware, lo cual establece el avance de la tecnología, que determinan fue el problema de la falta de desarrollo de la geografía en el siglo pasado. El libro incluye varios puntos de vista con referencias a la historia y a la visión de cómo debe de avanzar la geografía aplicada.

La obra no describe por completo los acontecimientos positivos y las limitaciones de la unión de diferentes disciplinas (la estandarización de los datos). El concepto de la geografía aplicada en Iberoamérica, que se muestra en este libro, presenta una imagen poderosa, contundente e incluyente. Los autores de los diferentes capítulos nos plantean un comienzo de una investigación muy prometedora, el avance en el conocimiento uniendo varias disciplinas. Las ideas que se plantean en esta obra resultan estimulantes para continuar indagando, y así obtener mejores respuestas a los siguientes cuestionamientos: ¿cuáles son los retos públicos a cubrir?, ¿cuáles son los problemas sociales a combatir? y ¿cuáles son los debates teóricos que se quedan en la agenda? 


\section{Fuentes consultadas}

Batty, Michel (2005), "Agents, cells, and cities: new representational models for simulating multiscale urban dynamics", Environment and Planning A, 37 (8), SAGE Publications, pp. 1373-1394.

Batty, Michel (2013), The new science of cities, Mit Press, Instituto Tecnológico de Massachusetts, Massachusetts.

Elwood Shannon, Michael Frank Goodchild y Daniel Sui (2012), "Researching volunteered geographic information: Spatial data, geographic research, and new social practice", Annals of the association of American geographers, 102 (3), Taylor and Francis, pp. 571-590.

Elwood Shannon, Michael Frank Goodchild y Daniel Sui (2013), "Prospects for VGI research and the emerging fourth paradigm", Crowdsourcing Geographic Knowledge, Springer, Netherlands, pp. 361-375.

Goodchild Michael Frank, Max J. Egenhofer, R. Fegeas, y Joan C. Kottman (eds.) (2012), Interoperating geographic information systems, vol. 495, Springer Science y Business Media.

Garrocho-Rangel, Carlos (2003), "La teoría de interacción espacial como síntesis de las teorías de localización de actividades comerciales y de servicios", Economía Sociedad y Territorio, IV (14), El Colegio Mexiquense A. C., Toluca, pp. 203-251.

Garrocho-Rangel Carlos y Gustavo D. Buzai (2015), Geografía Aplicada en Iberoamérica. Avances, retos y perspectivas, El Colegio Mexiquense A. C., Toluca.

Garrocho-Rangel, Carlos, Eduardo Jiménez-López y José Antonio Álvarez-Lobato, (2014), "Estructura profunda de los flujos migratorios en México, 1990-2010”, La situación demográfica en México 2014, Consejo Nacional de Población, México, pp. 87-118.

Heppenstall, Alison J., Andrew T., Crooks, Linda M. See y Michael Batty (eds.) (2011), Agent-based models of geographical systems, Springer Dordrecht Heidelberg, London-New York. 
Pírez, Pedro (2014), "La privatización de la expansión metropolitana en Buenos Aires", Economía Sociedad y Territorio, VI (21), El Colegio Mexiquense, A.C., Toluca, pp. 31-54.

Rey, Sergio J., y Luc Anselin, (2010), "PySAL: A python library of spatial analytical methods", Handbook of applied spatial analysis, Springer Berlin, Heidelberg, pp. 175-193.

Wilson, John P., y A. Stewart Fotheringham (eds.) (2008), The handbook of geographic information science, John Wiley \& Sons.

Recibido: 30 de agosto de 2016. Corregido: 14 de octubre de 2016. Aceptado: 1 de diciembre de 2016.

EDUARDO JIMÉNEZ-LÓPEZ EJIMENEZ@CMQ.EDU.MX El Colegio Mexiquense, A. C.

Eduardo Jiménez López. Mexicano. Doctor en ciencias aplicadas con especialidad en caos y sistemas dinámicos. Actualmente se desempeña como profesor-investigador en El Colegio Mexiquense, A. C. Sus líneas de investigación son geografía espacialmente integrada, análisis espacial y desarrollo urbano y regional. Entre sus publicaciones recientes destacan, en coautoría: "Estructura profunda de los flujos migratorios en México, 1990-2010", La situación demográfica en México 2014, Consejo Nacional de Población, México, pp. 87-118 (2014). "A family of hyperchaotic multi-scroll attractors in $\mathrm{R}^{\wedge} \mathrm{n}$ ", Applied Mathematics and Computation, 233 (1), Elsevier, pp. 522-533 (2014); "An approach to generate deterministic Brownian motion", Communications in Nonlinear Science and Numerical Simulation, 19 (8), Elsevier, pp. 2740-2746 (2014); "Generalized multistable structure via chaotic synchronization and preservation of scrolls", Journal of the Franklin Institute-Engineering and Applied Mathematics, 350 (10), Elsevier, pp. 2853-2866 (2013). 